\title{
Inserção do aluno de odontologia no SUS: contribuições do Pró-Saúde
}

Simone Dutra Lucas*, Andréa Clemente Palmier**, João Henrique Lara do Amaral***, Marcos Azeredo Furquim Werneck****, Maria Inês Barreiros Senna*****

\author{
* Professora adjunto da Faculdade de Odontologia da Universidade \\ Federal de Minas Gerais, doutora em saúde pública \\ ** Professora assistente da Faculdade de Odontologia da Universidade \\ Federal de Minas Gerais, doutoranda em saúde coletiva \\ *** Professor adjunto da Faculdade de Odontologia da Universidade \\ Federal de Minas Gerais, doutor em saúde coletiva \\ **** Professor associado da Faculdade de Odontologia da Universidade \\ Federal de Minas Gerais, doutor em odontologia social \\ ****** Professora adjunto da Faculdade de Odontologia da Universidade \\ Federal de Minas Gerais, doutora em educação
}

\section{RESUMO}

O objetivo deste trabalho é descrever a experiência da disciplina Ciências Sociais Aplicadas à Saúde (CSAS) da Faculdade de Odontologia da Universidade Federal de Minas Gerais (FO-UFMG). Em resposta às recomendações das Diretrizes Curriculares Nacionais para os cursos de graduação em Odontologia e do Programa Nacional de Reorientação da Formação Profissional em Saúde (Pró-Saúde), desde 2004, a FO-UFMG tem se mobilizado para mudar seu currículo, dando atenção especial à diversificação dos cenários de aprendizagem. Em 2007, a Disciplina de CSAS foi reformulada, permitindo a inserção dos discentes no Sistema Único de Saúde (SUS) no início de sua formação profissional, quando a realidade e a prática do SUS são os objetos do ensino. Esse movimento reforçou as expectativas de que essa inserção é viável. Espera-se que as mudanças na disciplina funcionem como um projeto piloto, subsidiando outras iniciativas que visem uma maior aproximação dos estudantes à prática profissional, e que sirva de parâmetro na organização e planejamento de outros conteúdos vinculados à saúde coletiva a serem incluídos na formação profissional.

\section{DESCRITORES}

Ciências Sociais. Currículo. Ensino. Odontologia. SUS.

o Brasil, o movimento de mudança na formação
profissional dos cirurgiões dentistas remonta aos anos 60. Foram realizados três seminários sobre o ensino odontológico na América Latina fomentados pela Organização Pan-americana da Saúde (OPAS), Fundação Kellogg e Associação Latinoamericana das Faculdades de Odontologia (ALAFO). Os seminários cumpriram seus propósitos, pois reuniram informações sobre as condições do ensino e mobilizaram alguns cursos de Odontologia no desenvolvimento de ações com o objetivo de inovar na formação dos recursos humanos na área da saúde. ${ }^{13}$ Nessa mesma década, e nas duas seguintes, o ensino odontológico foi marcado pelas propostas de modernização dos currículos dos cursos de graduação emanadas do Conselho Federal de Educação (CFE).${ }^{10}$ Entre elas destacam-se:

a) formação profissional para a realidade brasileira, b) perfil profissional generalista capaz de aplicar a filosofia preventiva e social aos problemas de saúde,

c) valorização e compreensão da participação popular e da abordagem multisetorial,

d) prática extramuros sob supervisão em instituições públicas com valorização da integração ensinoserviço. $^{7}$

No contexto das mudanças curriculares uma estratégia importante foi a articulação da formação profissional com as políticas de saúde e com o sistema de atenção à saúde. Buscava-se a integração ensinoserviços o que levou à adesão de escolas de Odontologia brasileiras ao Programa de Inovações de Ensino 
e Serviços Odontológicos, coordenado pela OPAS e Fundação KELLOG. Esse programa financiava experiências docente-assistenciais na América Latina com ênfase na simplificação da Odontologia. ${ }^{12}$

No campo da atenção à saúde, nas décadas de 70 e 80, o Brasil viveu o movimento da Reforma Sanitária culminando com a criação do Sistema Único de Saúde (SUS) que dentre as suas atribuições destaca-se o ordenamento da formação de recursos humanos para a área da saúde. ${ }^{3}$

Na educação superior, na década de 90 , foi promulgada a Lei de Diretrizes e Bases da Educação Nacional que estabeleceu o princípio da flexibilização curricular em substituição à rigidez dos currículos mínimos. O processo de discussão sobre as mudanças dos currículos mínimos dos cursos de graduação foi iniciado, em dezembro de 1997, pela Secretaria de Educação Superior (SESu) do Ministério da Educação com a abertura do edital ${ }^{\circ} 4 / 97$ para envio de proposta pelas Instituições de Ensino Superior (IES) para a elaboração das Diretrizes Curriculares Nacionais $(\mathrm{DCN}){ }^{11}$

As DCN para os cursos de graduação em Odontologia, aprovadas em 2002, apontam na direção da diversificação de cenários no SUS quando recomendam que a formação profissional deve incluir o sistema de saúde do país, a atenção integral à saúde e o trabalho em equipe. ${ }^{4} \mathrm{O}$ relatório da $3{ }^{\mathrm{a}}$ Conferência Nacional de Saúde Bucal sinaliza na mesma direção quando valoriza os convênios entre as instituições formadoras e os serviços de atenção à saúde bucal como oportunidade de aproximação dos estudantes dos modelos assistenciais e da realidade social da população. ${ }^{5}$

Um componente importante de mudança na formação depois da promulgação das DCN para a área da saúde é o Programa Nacional de Reorientação da Formação Profissional em Saúde (Pró-Saúde) do Ministério da Educação e Ministério da Saúde. Ele foi lançado em 2005, e tem como objetivo geral

\footnotetext{
"incentivar transformações do processo de formação, geração de conhecimentos e prestação de serviços à população, para abordagem integral do processo de saúde/doença". ${ }^{6}$
}

A Faculdade de Odontologia da Universidade Federal de Minas Gerais (FO-UFMG), a partir de 2004, em resposta às DCN e em sintonia com o PróSaúde, tem se mobilizado para uma alteração na sua matriz curricular. A perspectiva da mudança de acor- do com os princípios do programa está amparada na seleção da proposta do curso na sua primeira edição. Um dos objetivos propostos pela candidatura do curso ao Pró-Saúde é o fortalecimento e o avanço do seu processo de mudança curricular. A elaboração da nova matriz curricular tem considerado o perfil epidemiológico da população brasileira, a produção científica sobre as iniciativas de mudança na formação nas profissões da saúde, e as políticas para a educação e a saúde. A necessidade de ampla participação e co-responsabilização da comunidade universitária com a mudança curricular, as experiências acumuladas pelo curso, a busca de consensos, e a mudança na formação entendida como processo em que se reconhece os limites humanos e de infra-estrutura; são os outros elementos que têm balizado as proposições apresentadas pelo curso.

Nesse processo tem sido dada atenção especial à estratégia da diversificação dos cenários de aprendizagem uma vez que estes permitem uma aproximação dos estudantes das reais condições de saúde das comunidades. Os distintos cenários têm um potencial efeito indutor de transformação para o curso uma vez que revelam a contradição entre as condições sociais e os modelos de prática com enfoque predominante nos aspectos biológicos. Favorecem o desenvolvimento de uma formação mais crítica. O Pró-Saúde dá importância aos cenários de prática, não só pelas possibilidades de transformação apresentadas por essa proposta como pela demanda de acesso das populações beneficiárias dos programas e iniciativas das instituições formadoras de recursos humanos para a saúde.

O cenário de prática diz respeito,

\footnotetext{
“à incorporação e à inter-relação entre métodos didáticos, pedagógicos, áreas de prática e vivências, utilização de tecnologias e habilidades cognitivas e psicomotoras. Inclui também a valorização dos preceitos morais e éticos orientadores de condutas individuais e coletivas. Eles se relacionam também aos processos de trabalho, ao deslocamento do sujeito e do objeto do ensino e à revisão da interpretação das questões referentes à saúde e à doença, em que se considera sua dinâmica social". ${ }^{9}$
}

Dessa forma, o espaço físico, os equipamentos sociais e dos serviços, e os programas são condições necessárias para o desenvolvimento do ensino e da atenção à saúde. As instituições que participam da formação - universidade e serviço - imprimem um novo significado para a natureza e os conteúdos das 
práticas. Assim, supera-se a utilização dos serviços de caráter exclusivamente instrumental, fazendo dos cenários externos um prolongamento do espaço institucional da universidade. Trabalha-se também com a concepção de território que implica, além do componente geográfico, as condições de vida da população, perfil epidemiológico, acesso aos serviços de saúde, disponibilidade de equipamentos sociais, grau de mobilização e organização da população, e ações de caráter inter-setorial. Soma-se a essas possibilidades a formação no processo de trabalho da equipe multiprofissional. Propõe-se dessa forma responder propositivamente ao alerta de que na formação de recursos humanos a incerteza, os conflitos de valor e a singularidade das situações reais da prática não raras vezes escapam de soluções técnicas pré-definidas. ${ }^{14}$

Fundamentalmente, a intencionalidade primeira de como e porque se compartilham os cenários de aprendizagem com o serviço é a mudança no processo de formação profissional. O maior ou menor sucesso dessas iniciativas é diretamente dependente do quanto os sujeitos venham a se despir dos preconceitos ainda muito presentes nas relações interinstitucionais. Outro elemento de fundamental importância, nessa linha de trabalho, é dar oportunidade à inserção à prática desde o início do curso. O objetivo deve ser oferecer um significado necessário aos conteúdos curriculares tradicionalmente vinculados aos primeiros períodos da formação.

A reformulação da Disciplina de Ciências Sociais Aplicadas à Saúde (CSAS) foi uma das iniciativas do curso. Buscava-se a transformação de uma disciplina com enfoque eminentemente conceitual e fragmentado em sala de aula, para uma disciplina que apresentasse um eixo temático consistente e que envolvesse atividades práticas de inserção do discente no SUS no começo da formação profissional. Antes dessa mudança, os estudantes participavam de uma única visita às unidades básicas de saúde (UBS) no início das atividades clínicas do quarto período do curso. Uma segunda oportunidade de contato com o SUS, essa mais efetiva, consistia na participação dos estudantes no Estágio Supervisionado no último período da formação.

Originalmente, de 1982 até meados da década de 90, a disciplina de CSAS com conteúdos de Sociologia, Antropologia e Psicologia era ministrada por professores da área de ciências humanas, a saber, assistentes sociais, sociólogos e docentes da área da Psicologia. Ao longo do tempo, os conteúdos foram assumidos por uma cirurgiã-dentista com formação na área da Saúde Coletiva; em função da escassez destes profissionais na UFMG com disponibilidade para apoiar os cursos não vinculados às ciências humanas.

A decisão pela mudança dos conteúdos caminhou na direção de oferecer subsídios na área da Saúde Coletiva como aporte teórico capaz de iluminar experiências de inserção à prática no SUS e na comunidade. A disciplina busca enfatizar os problemas de saúde da população, a organização e estrutura disponíveis para a superação destas questões e o processo de trabalho que caracterizam o modelo de atenção à saúde vigente. Com a proposta de mudança foram incorporados docentes com formação em Saúde Coletiva. Há expectativa de que as alterações na disciplina funcionem como um projeto piloto subsidiando outras iniciativas. Pretende-se uma maior aproximação dos estudantes à prática profissional desde o início da formação. Esse movimento reforçou a idéia de que essas inserções são viáveis na proposta de mudança curricular do curso.

O novo projeto da disciplina foi elaborado por um grupo de trabalho formado por docentes com formação em saúde coletiva, sanitaristas da Prefeitura Municipal de Belo Horizonte, dentre eles os Coordenadores de Saúde Bucal, representantes do Centro de Educação em Saúde (CES) e gerentes de UBS.

O objetivo deste trabalho é sistematizar a experiência da FO-UFMG com a disciplina CSAS ministrada no segundo período do Curso de Odontologia relatando a inserção dos estudantes no SUS.

\section{MATERIAL E MÉTODOS}

A disciplina de CSAS é obrigatória, com uma carga horária de 45 horas. A equipe é composta por quatro docentes de dois departamentos da Unidade. O plano de ensino e o caderno de textos constituem o material didático disponibilizado. O plano de ensino apresenta as atividades que serão desenvolvidas ao longo do semestre, método, critérios de avaliação, roteiros para atividades práticas e bibliografia. $\mathrm{O}$ caderno de textos apresenta bibliografia básica e complementar.

Os objetivos da disciplina são conhecer e discutir as concepções do processo saúde/doença em distintos grupos sociais e compreender os determinantes sociais de saúde. Desse modo, o eixo estruturador da disciplina é o tema processo saúde/doença. Deste tema derivam outros conteúdos correlacionados.

A aprendizagem significativa observando as condições da aprendizagem de adultos, a utilização de metodologias ativas e a diversificação dos cenários de 
aprendizagem podem ser consideradas características da disciplina. As atividades com a participação dos estudantes acontecem em sala de aula e os cenários são o SUS e a comunidade.

A aprendizagem significativa trata da relação entre a nova informação e a estrutura de conhecimento do estudante, o que ela já conhece. ${ }^{8}$ No processo também são fundamentais a diversificação dos cenários, a construção permanente das informações e a valorização da prática. ${ }^{1} \mathrm{Na}$ disciplina fez-se a escolha pela problematização que consiste na aproximação crítica da realidade servindo-se dela como ocasião/desafio para a aprendizagem.

No campo das estratégias de ensino são utilizadas aulas expositivas dialogadas, estudo dirigido e de textos, atividades em grupos onde participa um docente tutor, atividade interativa nos cenários de prática e socialização de produtos. O tutor é aquele que acompanha e revê a prática discente e facilita o processo de ensino-aprendizagem, não qualquer processo, mas aquele centrado no estudante. Dele espera-se a competência para facilitar o processo do aprender a aprender e que tenha uma compreensão clara da prática desenvolvida pelos alunos. ${ }^{2}$

Não diferentemente de outras experiências a avaliação do processo de ensino aprendizagem é de difícil operacionalização. Nesse sentido, o corpo docente da disciplina tem trabalhado na busca de uma coerência cada vez maior entre os procedimentos de avaliação e os objetivos do ensino. Até o momento, a avaliação dos estudantes é feita no contexto de cada grupo. Eles são avaliados na produção escrita, na habilidade de exposição e análise das situações observadas, e na coerência das conclusões considerando o aporte trazido pela literatura. Na socialização dos produtos em sala de aula observa-se a clareza na exposição das idéias, a capacidade de síntese, e a organização lógica entre o registro das observações, a habilidade crítica e as conclusões.

A dinâmica da disciplina intercala as atividades de concentração em sala de aula com dispersão nos cenários.

Durante as atividades de concentração, em sala de aula, busca-se a motivação dos estudantes para a construção do conhecimento científico dos temas abordados considerando as experiências do cotidiano e a prática social. É feito um levantamento das representações dos discentes sobre os conteúdos curriculares, entre eles: processo saúde-doença e o SUS. A seguir, faz-se o estudo dirigido da literatura disponível e a problematização das representações do grupo. Artigos científicos e textos oficiais são utilizados nessa fase. A discussão é feita em grupos de até vinte estudantes.

O passo seguinte é a preparação dos alunos para o desenvolvimento das atividades nas UBS. Neste sentido, foram elaborados roteiros, previamente testados, sobre cada conteúdo objeto das práticas nos cenários. Após cada atividade no campo de prática os estudantes elaboram uma síntese entre as impressões previamente construídas e o que foi observado nos cenários. Estimula-se, com isso, que os discentes possam identificar as maiores ou menores aproximações entre as primeiras elaborações construídas pelo grupo e a prática social vigente. Desse modo, a realidade torna-se mediadora da aprendizagem.

Tendo em vista os objetivos da disciplina trabalham-se as seguintes temáticas:

- o conceito de saúde dos usuários e trabalhadores do SUS;

- o acolhimento, a visita domiciliar, o processo de trabalho e a gestão local nas UBS;

- os recursos materiais e humanos; e

- a infra-estrutura da atenção à saúde.

As atividades são desenvolvidas em dez UBS localizadas em três Distritos Sanitários apoiadas pelo gestor local e trabalhadores. Durante as visitas domiciliares, os alunos são acompanhados pelos Agentes Comunitários de Saúde (ACS). As ações compreendem a realização de uma enquete sobre o conceito de saúde dos diferentes sujeitos, a coleta de informações sobre a gestão e o processo de trabalho, o registro da estrutura física, dos recursos materiais, humanos, a participação nas atividades de acolhimento e a realização de visitas domiciliares.

Nas UBS os estudantes são acompanhados por profissionais dos serviços. Na educação médica, a figura do profissional que auxilia na formação tem recebido diferentes denominações entre elas supervisor, mentor, preceptor e tutor. ${ }^{2} \mathrm{O}$ esforço dos autores culminou com uma proposta de padronização na utilização desses termos que a nosso ver não consegue responder ao perfil da atividade do profissional do serviço no caso específico da disciplina. Entretanto, oferece elementos suficientes para inicialmente situar essa atuação no conjunto das ações que se espera de um preceptor, quais sejam a de dar suporte, compartilhar experiências, reduzir a distância entre a teoria e prática favorecendo a execução das ações durante as atividades de ensino. 


\section{RESULTADOS}

A experiência com a disciplina tem mostrado que apenas um número reduzido dos estudantes se considera usuários dos serviços de atenção à saúde, por não compreenderem a diversidade de ações do sistema. Os alunos, na sua maioria, não projetam o serviço público como espaço preferencial para a atuação como profissionais de saúde. As informações que detêm sobre o SUS são aquelas veiculadas pela mídia. Após o reconhecimento dos cenários de prática as impressões anteriormente registradas sobre o SUS começam a ser substituídas por percepções mais positivas, e de surpresa diante do que foi observado.

A concepção de saúde como perfeito estado de equilíbrio das funções, órgãos e sistemas como normalidade fisiológica e mental prevalece entre os estudantes. A doença é percebida como um rompimentodesseestadodeequilíbrio. Osestudantesidentificam diferentes percepções do processo saúde/doença entre os trabalhadores da UBS e usuários. O mesmo acontece entre diferentes categorias profissionais. De forma bastante particular os usuários das UBS relacionam a doença com a falta de condições para o trabalho. Quanto à saúde bucal os discentes se surpreendem com o fato de algumas pessoas relatarem ausência de doença bucal mesmo entre aquelas que afirmam já ter se submetido a alguma intervenção odontológica. Esta atividade propicia reconhecer diferentes formulações do conceito saúde/doença nas diferentes classes sociais permitindo compreender os significados da determinação social da saúde. Buscase que o aluno também se situe no contexto de sua origem social e reflita sobre o que tem determinado a formulação do próprio conceito de saúde/doença.

A participação no acolhimento e as visitas domiciliares colocam os estudantes em contato com atividades extremamente importantes à percepção dos tipos de problemas e demandas da população assim como o seu enfrentamento diante da capacidade de resposta do SUS. O acolhimento desnuda a complexidade do acesso aos serviços de saúde, as diferentes demandas dos usuários e trás à tona a questão das condições agudas. Mostra também aspectos do processo de trabalho não reconhecidos pelo estudante permitindo o exercício da dúvida em relação aos papéis a serem desempenhados por diferentes profissionais. As visitas domiciliares, além de revelar a estratégia não reconhecida pela maioria dos estudantes onde profissionais do serviço se deslocam até a residência das famílias, permite a constatação, in loco, das condições de vida e sua relação com as condições de saúde. As duas experiências revelam a importância da compreensão de que, para além da biologia humana, as condições de vida das pessoas, valores e hábitos, estilos de vida e ambiente, e a organização política e social interferem diretamente na situação de saúde da população. Introduz-se, assim, um conceito absolutamente novo para os estudantes: o da determinação social da saúde.

\section{DIscussão}

O caminho metodológico proposto pela disciplina atende aos pressupostos das DCN e aos princípios do Pró-Saúde de produzir uma aprendizagem que tenha a realidade e a prática do SUS como objetos do ensino. Vivenciar o processo de trabalho das UBS propicia o surgimento, para o estudante, de um conceito de trabalho que supera a ação centrada no profissional, na consulta clínica odontológica, na prescrição de medicamentos e solicitação de exames complementares. Permite a incorporação de outros educadores, no processo de formação, como os profissionais ligados aos serviços. Possibilita, também, o desenvolvimento da capacidade crítica do estudante de Odontologia facilitando a reformulação de conceitos e a conseqüente criação de novos conhecimentos.

Quanto aos atores do serviço de saúde, estes atribuem importância à inserção dos futuros profissionais no SUS, mesmo no início do Curso de Odontologia quando as suas contribuições são limitadas; por compreenderem que se pode formar um profissional mais capacitado para atuar na saúde pública.

O Pró-Saúde potencializou os esforços que vinham sendo implementados com o objetivo de promover a mudança da matriz curricular na FO-UFMG. No caso específico da disciplina de CSAS houve um fortalecimento das ações e motivação dos professores com pronta resposta da parte da Coordenação de Saúde Bucal do Município de Belo Horizonte. A abertura dos novos cenários de prática para a disciplina foi pactuada no contexto e desdobramentos do PróSaúde incluindo a realocação e aquisição de equipamentos para os cenários do Estágio Supervisionado do nono período do curso. Outro avanço importante foi a mudança da disciplina de CSAS do segundo para o primeiro período na nova matriz curricular atendendo à expectativa de inserção dos estudantes à prática desde o início da formação. Mais imediatamente, a experiência da disciplina de CSAS servirá de parâmetro na organização e planejamento de outros conteúdos vinculados à saúde coletiva a serem incluídos na formação profissional. 


\section{CONCLUSÕES}

Considera-se que a experiência da disciplina incorpora os três eixos do Pró-Saúde:

a) orientação teórica devido ao seu referencial teórico que é o processo saúde/doença;

b) cenários de prática pela integração ensino-serviço e diversificação dos cenários de aprendizagem e

c) orientação pedagógica por meio da adoção de metodologias ativas de ensino-aprendizagem e pela análise crítica da atenção primária.

\section{ABSTRACT}

\section{Insertion of the dental student in the SUS: contributions of the Pro-Health program}

The aim of this study was to describe the experience of the Social Sciences Applied to Health (CSAS) discipline of the Dental School of the Federal University of Minas Gerais (FO-UFMG). In response to the National Curriculum Guidelines for the dental undergraduate course and the National Program of Reorientation of Health Professional Training (PróSaúde), the FO-UFMG has been undergoing curriculum reform since 2004. Special attention has been given to the range of scenarios in the teaching process. In 2007, the CSAS discipline was changed, allowing the insertion of the students into the Unified Health System (SUS) as of the beginning of their training, when reality and SUS practice are the teaching subjects. This movement reinforced the expectation that this insertion is viable. It is hoped that the changes in the discipline will be used as a pilot project, supporting other initiatives that aim at putting students closer to professional practice and and that serve as a model in the organization and planning of other subjects related to public health, to be ultimately included in professional training.

\section{DESCRIPTORS}

Social Sciences. Curriculum. Teaching. Dentistry. Unified Health System (SUS). •

\section{REFERÊNCIAS BIBLIOGRÁFICAS}

1. Batista $\mathrm{N}$ et al. $\mathrm{O}$ enfoque problematizador na formação de profissionais da saúde. Rev Saúde Pública 2005; 39 (2) 231-237.

2. Botti SHO, Rego S. Preceptor, supervisor, tutor e mentor: quais são seus papéis? Rev Bras Educ Med 2008; 32(3) 363-373.

3. BRASIL. Lei n. 8080 - 19 set. 1990. Dispõe sobre as condições para a promoção, proteção e recuperação da saúde, a organização e o funcionamento dos serviços correspondentes, e dá outras providências. Diário Oficial, Brasília, 20 set. 1990.
4. BRASIL. Ministério da Educação. Conselho Nacional de Educação / Câmara de Educação. Resolução CNE/CES nº 3/2002, de 19 de fevereiro de 2002. Institui Diretrizes Curriculares Nacionais do Curso de Graduação em Odontologia. Brasília, DF, 19 fev. 2002.

5. BRASIL. Ministério da Saúde. Conselho Nacional de Saúde. $3^{\text {a }}$ Conferência Nacional de Saúde Bucal, Brasília, DF, de 29 de julho a $1^{\circ}$ de agosto de 2004 - Relatório Final. Disponível em: http://www.saude.sc.gov.br/Eventos/ConferenciaSaudeBucal/relatorio_nacional.pdf

6. BRASIL. Ministério da Saúde. Ministério da Educação. Programa Nacional de Reorientação da Formação Profissional em Saúde - Pró-Saúde. Série C. Projetos, Programas e Relatórios. Brasília, DF, 2005.

7. Conselho Federal de Educação. Resolução no. 04, de 03 de setembro de 1982. Fixa os mínimos de conteúdo e duração do curso de Odontologia. Ministério da Educação e Cultura. Diário Oficial, 16/09/1982

8. Dellaroza MSG, Vannuchi MTO. (Org.). O currículo integrado do Curso de Enfermagem da Universidade Estadual de Londrina: do sonho à realidade. São Paulo: HUCITEC, 2005.

9. Feuerwerker LCM, Costa H, Rangel ML. Diversificação de cenários de ensino e trabalho sobre necessidades/problemas da comunidade. Divulgação em Saúde para Debate 2000; (22) 36-48.

10. Lucas SD. Formação profissional de cirurgiões-dentistas egressos de dois cursos superiores com orientações distintas. Belo Horizonte, 1995. Mestrado [Dissertação]. Faculdade de Educação da Universidade Federal de Minas Gerais.

11. Martins RO. Permanência e movimento: um olhar sobre o processo e construção das diretrizes curriculares para o ensino superior no contexto das políticas do MEC. Brasília, 2004. Tese [Doutorado em Sociologia]. Instituto de Ciências Sociais da Universidade de Brasília.

12. Mendes EV, Marcos B. Odontologia integral: bases teóricas e suas implicações no ensino e na pesquisa odontológicas. Belo Horizonte: PUCMG/FINEP, 1985.

13. Queiroz MG, Dourado LF. O ensino da odontologia no Brasil: uma leitura com base nas recomendações e nos encontros internacionais da década de 1960. Hist. cienc. saude-Manguinhos 2009; 16(4) 1011-1026.

14. Schön DA. Educando o profissional reflexivo: um novo design para o ensino e a aprendizagem. Porto Alegre: Artmed, 2000.

Recebido em 07/07/2011 Aceito em 25/07/2011 